\title{
Movement disorders associated with chromosomal aberrations diagnosed in adult patients
}

\author{
Monika Figura ${ }^{1}$, Maciej Geremek ${ }^{2}$, Łukasz M. Milanowski ${ }^{1,3}$, Izabela Meisner-Kramarz ${ }^{1}$, \\ Karolina Duszyńska-Wąs ${ }^{1}$, Stanisław Szlufik ${ }^{1}$, Dorota Różański ${ }^{1}$, Marta Smyk ${ }^{2}$, Dariusz Koziorowski ${ }^{1}$ \\ ${ }^{1}$ Department of Neurology, Faculty of Health Sciences, Medical University of Warsaw, Poland \\ ${ }^{2}$ Institute of Mother and Child, Department of Medical Genetics, Warsaw, Poland \\ ${ }^{3}$ Department of Neurology, Mayo Clinic, Jacksonville, Florida, USA
}

\begin{abstract}
Introduction. Chromosomal aberrations are rare but important causes of various movement disorders. In cases of movement disorders associated with dysmorphic features, multiorgan involvement and/or intellectual disability, the identification of causative chromosomal aberrations should be considered.

Aim of the study. The purpose of this article was to summarise clinical findings in six patients with dystonia and two with parkinsonism and identified chromosomal aberrations in a single-centre prospective study.

Materials and methods. 15 adult patients with dystonia or parkinsonism were referred to array comparative genomic hybridisation (aCGH) testing from our Department of Neurology between 2014 and 2019. Additionally, one patient had a karyotype examination. Detailed clinical, psychological and radiological diagnostics were performed in each case.

Results. Chromosomal aberrations were identified in six patients with dystonia and two with parkinsonism. Two patients were identified with aberrations associated with de Grouchy syndrome. We also reported generalised dystonia in patients with deletion in $3 q 26.31$ and duplication in 3p26.3, as well as dystonia and hypoacusis in a patient with duplication in Xq26.3. One patient was diagnosed with duplication in 21q21.1. Early-onset parkinsonism was a manifestation of deletion in the 2q24.1 region. Late onset parkinsonism was also present in the patient with the most severe aberrations (duplication 1q21.1q44; deletion 10p15.3p15.1; deletion 10q11.21).

Conclusions. Dystonia and parkinsonism are possible manifestations of chromosomal aberrations. Chromosomal aberrations should be excluded in patients with early-onset movement disorders and concomitant dysmorphic features and/or intellectual disability. It is important to include this cause of movement disorders in future classifications. aCGH can be a valuable diagnostic tool in the evaluation of movement disorder aetiology.
\end{abstract}

Key words: dystonia, Parkinson's disease, chromosomal aberrations, microarray, parkinsonism

(Neurol Neurochir Pol 2021; 55 (3): 300-305)

\section{Introduction}

Dystonia and parkinsonism are among the most commonly diagnosed movement disorders. There is, however, no specific mention of chromosomal aberrations as a cause of dystonia in the IPDMS dystonia classifications [1,2]. In parkinsonism, a genetic background is usually observed in those patients with disease onset before the age of 50 or with a positive family history $[3,4]$.
Structural aberrations are caused by a rearrangement of the genetic material. They can be associated with a very broad clinical spectrum, from asymptomatic or benign to lethal. The study by Dale et al. suggested that up to $28 \%$ of children with movement disorders of suspected genetic aetiology have chromosomal abnormalities [5].

Chromosomal aberrations in selected movement disorder patients are important to identify. They cause multiorgan

Address for correspondence: Dariusz Koziorowski, Department of Neurology, Faculty of Health Sciences, Medical University of Warsaw, Kondratowicza 8 Str., 03-242 Warsaw, Poland, e-mail: dkoziorowski@wum.edu.pl 
involvement, and extended diagnostic measures need to be undertaken in order to exclude cardiac and endocrine system abnormalities. Assessment of chromosomal imbalances may be performed using standard karyotyping or molecular diagnostics with array comparative genomic hybridisation (aCGH).

aCGH is based on identifying differences in fluorescence signal intensity between the patient and reference DNA samples hybridised to the array. Its main limitation is an inability to detect balanced aberrations. It has a higher resolution of $\sim 100 \mathrm{kbp} v s .5 \mathrm{Mbp}$ for karyotype examination.

An American expert consensus statement from 2010 identified aCGH as a first-line diagnostic tool for the postnatal testing of patients with autism-spectrum disorder, intellectual disability, and multiple congenital anomalies [6]. aCGH is often performed in children with developmental delays, epilepsy or multiorgan manifestations, but rarely in adult patients with movement disorders. Some reports have suggested that it may be of value, particularly when atypical features appear in patients with otherwise acknowledged syndromes. This includes the paper by Lohmann et al. on the identification of causes of dopa-responsive dystonia and eye and skeletal abnormalities in a large family, in which aCGH examination revealed deletion on chromosome 6, including the CGH1 gene [7]. Similarly, a case of brain-lung-thyroid syndrome was described in a girl with additional immunodeficiency. Negative PCR and Sanger sequencing led to an aCGH examination, where the deletion of 3.32 Mbp in the chromosome 14q13.2-q21.1 region was detected, including genes NKX2-1 but also genes involved in immunological response [8].

A few other studies have reported chromosomal aberration as a potential cause of early-onset PD. An aCGH study in patients with early-onset levodopa-responsive parkinsonism and intellectual disability revealed partial trisomy in $4 \mathrm{q}$ [9]. The best-characterised chromosomal aberration associated with $\mathrm{PD}$ is 22q11 deletion. The study by Mok et al. revealed eight carriers from a total cohort of 9,387 PD patients confirmed by aCGH examination [10]. In a recent publication, an aCGH examination revealed cases with duplication of the $S N C A$ gene and heterozygous intragenic deletion of the GBA gene in $99 \mathrm{PD}$ subjects with a positive family history [11].

In our paper, we describe a cohort of adult patients with neurodevelopmental disorders and concomitant dystonia or parkinsonism and chromosomal aberrations.

\section{Materials and methods}

We prospectively identified and examined patients hospitalised in the Department of Neurology between 2006 and 2019 in whom dystonia or parkinsonism caused by karyotype abnormalities were suspected. Early onset of neurological symptoms, the progressive nature of symptoms, neurodevelopmental delay or negative metabolic investigations led to aCGH or karyotype examinations. Fifteen adult patients with dystonia or parkinsonism were referred to cytogenetic testing from our Department of Neurology between 2014 and 2019. In 7/15 patients, aCHG testing indicated pathogenic aberrations or variants of uncertain significance. We also included in our study one patient with significant karyotype findings from an examination performed in 2006. Each patient with chromosomal aberration underwent comprehensive clinical, psychological and radiological evaluations. The Polish version of MDS-UPDRS was performed in parkinsonian patients [12].

aCGH was performed using a $60 \mathrm{~K}$ oligonucleotide microarray (CytoSure, ISCA v3 Oxford Gene Technology, Oxford, UK). The patient's DNA was hybridised against the control DNA. Labelling and hybridisation were performed following the manufacturer's protocols. $500 \mathrm{ng}$ of DNA was labelled and purified on the column's centrifugal filters. After probe denaturation and prehybridisation with Cot-1 DNA, hybridisation was performed at $65^{\circ} \mathrm{C}$ with rotation for $20 \mathrm{hrs}$. After washing, the array was analysed with Agilent scanner and Feature Extraction software (Agilent Technologies, Santa Clara, CA, USA) and text file outputs from the quantisation analysis were imported to CytoSure Interpret software (Oxford Gene Technology) for copy number analysis.

This study was approved by the Ethics Committee of the Medical University of Warsaw (KB/56/2018) and has been performed in accordance with the ethical standards laid down in the 1964 Declaration of Helsinki and its later amendments. All participants signed an informed consent form for genetic examination prior to their inclusion in the study.

\section{Results}

Patients with CNV and karyotype aberrations were analysed in detail. Family history of dystonia or parkinsonism was negative in all cases. All the dystonia patients had been born from uneventful pregnancies and 5/6 received 10-point Apgar scores. In all patients, the onset of dystonia was in childhood. The pattern of the progression of dystonia was highly variable but in most (5/6) cases it led to generalised dystonia. The key clinical features we identified in our cohort, apart from dystonia, were dysmorphic features of the face, which were present in five out of six patients (Patients 1-5), hearing impairment in two (Patients 4 and 6), and epilepsy in one (Patient 2). The pattern of dystonia involvement varied greatly among patients, from focal foot dystonia to severe generalised dystonia affecting speech and walking. Patients from the PD group had later manifestation of movement disorder than did those in the dystonia group, developing their first parkinsonian symptoms when aged 37 (Patient 7) and 62 (Patient 8). Both had good responses to levodopa treatment without motor fluctuations and dyskinesia. The clinical characteristics of all patients are set out in Table 1.

Detailed psychological assessment revealed intellectual disability in most of the patients, regardless of the type of movement disorder. Intellectual disability varied from mild to severe. 


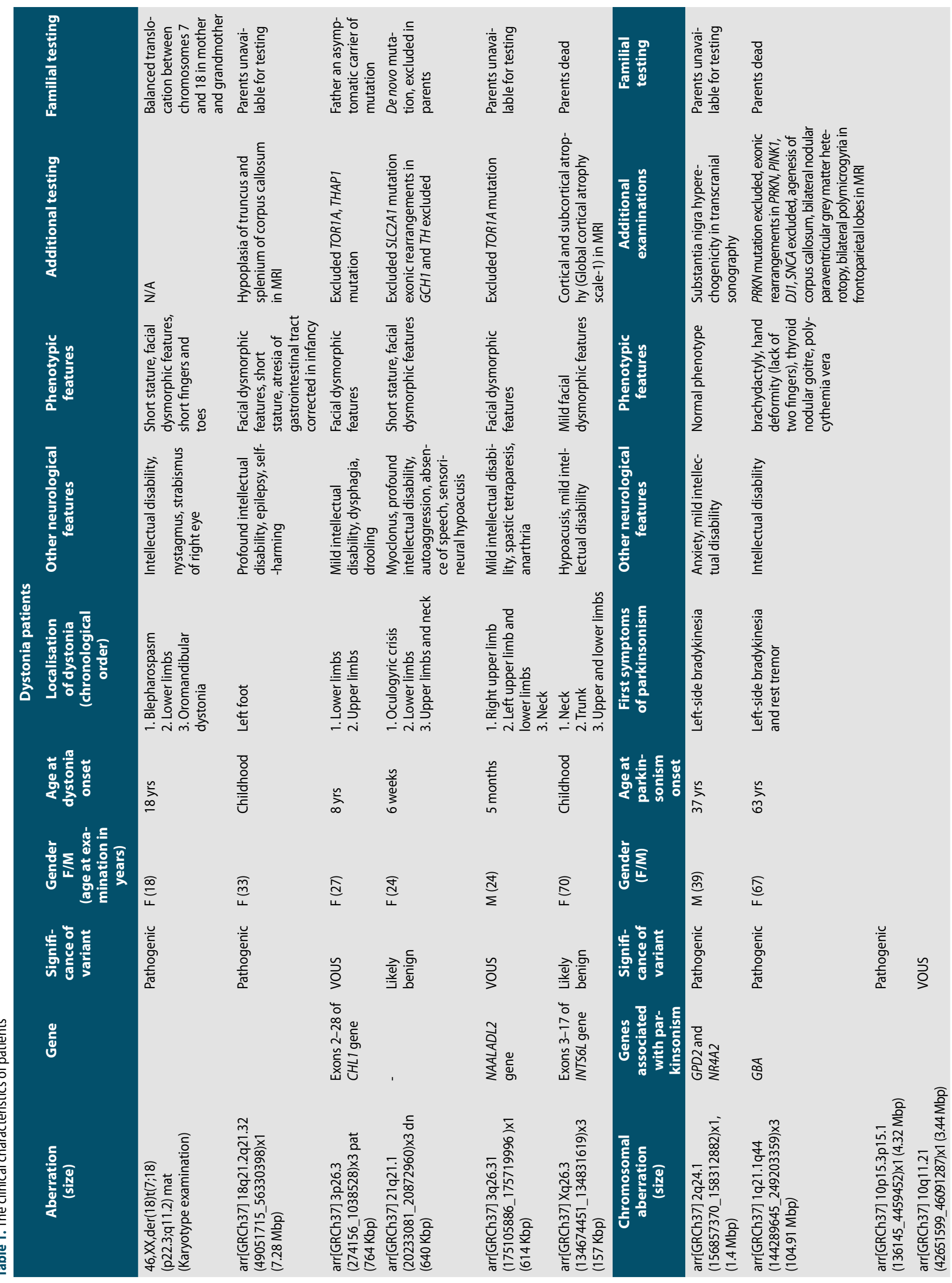


All patients underwent magnetic resonance imaging (MRI) studies. Some patients underwent other genetic testing prior to aCGH. In three cases, family members also underwent aCGH. In other cases, the parents were lost to follow-up. Outcomes of these examinations are summarised in Table 1.

Genetic imbalance previously associated with dystonia was found in two patients (Patients 1 and 2). Patient 7 was diagnosed with deletion involving GPD2 and NR4A2 genes, previously discussed in the pathology of PD.

Genetic findings in Patients 3, 4, 5, 6 and 8 were variants of uncertain clinical significance. In Patient $3, \mathrm{aCGH}$ examination revealed duplication involving exons 2-28 of the CHL1 gene. In Patient 4, duplication in the long arm of chromosome 21 did not include any protein-coding genes. Patient 5 was diagnosed with a deletion involving part of the NAALADL2 gene. He was affected by severe dystonia and intellectual disability. Duplication of exons 3-17 of the INTS6L gene was identified in Patient 6, who was suffering from dystonia, hearing impairment and intellectual disability. Patient 8 had the most severe aberrations. All the findings in karyotype and aCGH examinations are summarised in Table 1.

The remaining patients with dystonia or parkinsonism who underwent aCGH in our Department had normal results.

\section{Discussion}

Our study indicates that patients with movement disorders and genetic imbalance are a very heterogeneous group in terms of clinical manifestation.

We present eight patients with complex neurological symptoms, including early-onset dystonia or parkinsonism. Vast differential diagnosis was performed in each case. Another important feature present in the majority of patients was intellectual disability of varying degree. In some cases, chromosomal aberrations included genes previously associated with dystonia or parkinsonism which may have effects similar to monogenic disorders. The co-occurrence of the symptoms of movement disorders, intellectual disability and dysmorphic features, after exclusion of common genetic causes, led to testing for chromosomal abnormalities.

Two patients in our cohort (Patients 1 and 2) were diagnosed with De Grouchy syndrome (deletion in the $18 \mathrm{p}$ or $18 \mathrm{q}$ region). 18p deletion (historically known as De Grouchy syndrome type 1) is a recognised cause of dystonia as well as other movement disorders, including tics, myoclonus and ataxia. The incidence is estimated to be about 1:50,000 liveborn infants [13]. The syndrome is characterised by short stature, developmental delay and dysmorphic features - all of which were present in our patients. It is considered that epilepsy appears more often in cases of the $18 \mathrm{q}$ syndrome than the $18 \mathrm{p}$ syndrome, which is consistent with our observations. Dystonia is much less frequent in the $18 \mathrm{q}$ syndrome, with only anecdotal reports [14].
An absence of the GNAL gene may contribute to dystonia in patients with the $18 \mathrm{p}$ deletion syndrome. However, the frequency of dystonia in 18p deletion patients involving GNAL has been estimated at only $3 \%$ [15].

The other patients in our group (Patients 3 and 4) were diagnosed with chromosomal aberrations in which no linkage to dystonia or parkinsonism has been made so far. The significance of these findings is therefore uncertain but, in the absence of other causes, possible. This is also supported by the presence of the additional features of hearing impairment, dysmorphic facial features and intellectual disability, strongly suggesting the clinical importance of the detected aberrations.

Patient 3 was diagnosed with a microduplication of the CHL1 gene. This aberration has already been reported in the literature in patients with neurodevelopmental delay, learning and language difficulties, and seizures. Mild dysmorphic features and autism spectrum disorder were also described. To the best of our knowledge, neither dystonia nor other movement disorders have been described among patients with CHL1 duplication. Interestingly, Patient 3's father was an asymptomatic carrier of the mutation.

In one patient (Patient 4) pronounced myoclonus, hearing deficits, dysmorphic facial features, intellectual disability and psychiatric symptoms were also observed. Duplication in the long arm of chromosome 21 did not encompass regions with known genes, therefore this CNV is most likely benign. We can however speculate, as several long non-coding RNA are encoded in that region, that these could play a role in disease mechanisms [16].

Patient 5 was diagnosed with partial deletion in the long arm of chromosome 3, which included the NAALADL2 gene. A breakpoint of de novo balanced translocation has been mapped to that gene in a Cornelia de Lange $(\mathrm{CdL})$ syndrome patient. However, further sequencing and screening of the gene did not reveal any pathogenic variants in a cohort of patients with CdL syndrome [17]. Our patient had dysmorphic craniofacial features which were inconsistent with the CdL phenotype (steeple skull, high-arched palate), intellectual disability, and speech problems. There was no involvement of other organs.

Patient 6 (a female) had a duplication on chromosome X. The duplicated region is not located in a pseudoautosomal region, and therefore this raises a question as to whether $\mathrm{X}$ chromosome inactivation could have an effect on clinical expression. In some cases involving larger duplications on the $\mathrm{X}$ chromosome, a non-random, skewed preferential inactivation of the aberrant $\mathrm{X}$ chromosome has been observed. However, several cases of smaller duplications have been reported where the normal X chromosome was preferentially inactivated, leading to severe phenotype [18]. The X chromosome inactivation process may lead to a mosaic distribution of the active region, harbouring the duplication within the body, organs and tissues. The $\mathrm{X}$ chromosome inactivation status of the specific brain areas should be considered. A brain tissue sample was not available for inactivation testing. Madrigal et al. 
described two patients with partial duplications involving the long arm of the $\mathrm{X}$ chromosome and intellectual disability, short stature, microcephaly and hypopituitarism among others [19].

The deletion identified in Patient 7 contains two protein-coding genes: NR4A2 and GPD2. GPD2 encoding mitochondrial glycerophosphate dehydrogenase has not been unambiguously linked to any movement or neurodevelopmental disorder. Deletions encompassing NR4A2 alone, or with GPD2, have been found in a few patients with intellectual disability, epilepsy, language impairment and/or dysmorphic features. Recently, two patients have been reported with loss-of-function variants in NR4A2 and mild intellectual disability with dystonia-parkinsonism in early adulthood [20]. On the molecular level, the haploinsufficiency caused by deletion in our patient should result in a dosage effect similar to the frameshift mutations in the two patients described recently [20]. This case further strengthens the hypothesis that parkinsonism is a part of the clinical picture associated with NR4A2 dysfunction.

Patient 8 had the most severe aberrations, including many genes which could be responsible for parkinsonism. The patient had a $104 \mathrm{Mbp}$ duplication on chromosome 1 containing more than 1,000 genes, a 34-gene containing deletion encompassing 4.32 Mbp of $10 \mathrm{p}$, and a $3.44 \mathrm{Mbp}$ deletion on $10 \mathrm{q}$ containing 46 genes. The patient's aberration on chromosome 1 resulted in a duplication of the $G B A$ gene region. Heterozygous point mutations are a known parkinsonism risk factor, and homozygotes present with the more severe Gaucher disease. Some clinical features such as severe depression and dementia are consistent with typical GBA-PD patients. However, full GBA gene deletions/duplications have not been described in PD. Copy number variants in the GBA gene have been identified in five parkinsonian patients, although none of them had a full gene duplication [21].

\section{Conclusions}

Chromosomal aberrations may be an under-recognised cause of movement disorders. When dystonia or parkinsonism is the dominant clinical manifestation, karyotype examination may not be considered early. In cases when other features dominate, including neurodevelopmental problems, autism spectrum disorder, epilepsy or dysmorphic features, aCGH examination is prioritised. It is nevertheless important to keep in mind that karyotype studies may serve as an important first-line diagnostic tool in patients with early-onset movement disorders. In selected cases, such patients may require an examination of the cardiac system, a hearing examination and a gastrological assessment to exclude possible comorbidities caused by chromosomal aberrations.

We have identified some rare aberrations that have not been previously associated with parkinsonism or dystonia. They represent variants of uncertain clinical significance with possible association with the disorder. The lack of functional studies and whole-exome sequencing in patients is a limitation of our study. However, in all cases the general clinical picture and neurological symptoms, as well as an absence of the variant in some patient's parents and gene content, suggest their importance. In $4 / 8$ patients, aberrations contained genes in which mutations have been previously associated with dystonia or parkinsonism. Further studies are needed in the remaining cases to investigate whether they play a pathogenic role in the disease process.

Chromosomal abnormalities should be mentioned along with monogenic autosomal dominant and recessive causes of common movement disorders. Chromosomal aberrations may produce the same effect on gene expression and result in clinical phenotypes of monogenic disorders. It is known that small deletions and duplications ( $1 \mathrm{kbp}$ to $10 \mathrm{Mbp}$ ) constitute up to $15 \%$ of all mutations underlying human monogenic diseases [22].

In this burgeoning era of precision medicine and vector-based therapies for the treatment of $\mathrm{PD}$ and dystonia, it is increasingly important to identify disease backgrounds [23].

Therefore, we recommend the introduction of an aCGH examination as an important and cost-effective diagnostic tool, especially in cases of early-onset dystonia and selected cases of parkinsonism with intellectual disability in adult patients.

Funding: This study was funded from statutory funds of the Department of Neurology, Faculty of Health Science, Medical University of Warsaw. L.M. is supported by the Polish National Agency for Academic Exchange Iwanowska's Fellowship PPN/ IWA/2018/1/00006/U/00001/01.

Conflicts of interest: None.

\section{References}

1. Albanese A, Bhatia K, Bressman SB, et al. Phenomenology and classification of dystonia: a consensus update. Mov Disord. 2013; 28(7): 863-873, doi: 10.1002/mds.25475, indexed in Pubmed: 23649720.

2. Albanese A, Asmus F, Bhatia KP, et al. EFNS guidelines on diagnosis and treatment of primary dystonias. Eur J Neurol. 2011; 18(1): 5-18, doi: 10.1111/j.1468-1331.2010.03042.x, indexed in Pubmed: 20482602.

3. Berardelli A, Wenning GK, Antonini A, et al. EFNS/MDS-ES recommendations for the diagnosis of Parkinson's disease. European Journal of Neurology. 2012; 20(1): 16-34, doi: 10.1111/ene.12022.

4. Yoon WT. Comparison of dystonia between Parkinson's disease and atypical parkinsonism: The clinical usefulness of dystonia distribution and characteristics in the differential diagnosis of parkinsonism. Neurol Neurochir Pol. 2018; 52(1): 48-53, doi: 10.1016/j. pjnns.2017.11.004, indexed in Pubmed: 29196058.

5. Dale RC, Grattan-Smith P, Nicholson M, et al. Microdeletions detected using chromosome microarray in children with suspected genetic movement disorders: a single-centre study. Dev Med Child Neurol. 2012; 54(7): 618-623, doi: 10.1111/j.1469-8749.2012.04287.x, indexed in Pubmed: 22515636.

6. Miller DT, Adam MP, Aradhya S, et al. Consensus statement: chromosomal microarray is a first-tier clinical diagnostic test for individuals 
with developmental disabilities or congenital anomalies. Am J Hum Genet. 2010; 86(5): 749-764, doi: 10.1016/j.ajhg.2010.04.006, indexed in Pubmed: 20466091.

7. Lohmann K, Redin C, Tönnies H, et al. Complex and Dynamic Chromosomal Rearrangements in a Family With Seemingly Non-Mendelian Inheritance of Dopa-Responsive Dystonia. JAMA Neurol. 2017; 74(7): 806-812, doi: 10.1001/jamaneurol.2017.0666, indexed in Pubmed: 28558098.

8. Villafuerte B, Natera-de-Benito D, González A, et al. The Brain-Lung-Thyroid syndrome (BLTS): A novel deletion in chromosome 14q13.2-q21.1 expands the phenotype to humoral immunodeficiency. Eur J Med Genet. 2018; 61(7): 393-398, doi: 10.1016/j. ejmg.2018.02.007, indexed in Pubmed: 29477862.

9. Garraux G, Caberg JH, Vanbellinghen JF, et al. Partial trisomy $4 q$ associated with young-onset dopa-responsive parkinsonism. Arch Neurol. 2012; 69(3): 398-400, doi: 10.1001/archneurol.2011.802, indexed in Pubmed: 22410449.

10. Mok KY, Sheerin U, Simón-Sánchez J, et al. International Parkinson's Disease Genomics Consortium (IPDGC). Deletions at 22q11.2 in idiopathic Parkinson's disease: a combined analysis of genome-wide association data. Lancet Neurol. 2016; 15(6): 585-596, doi: 10.1016/S1474-4422(16)00071-5, indexed in Pubmed: 27017469.

11. Robak LA, Du R, Yuan Bo, et al. Integrated sequencing and array comparative genomic hybridization in familial Parkinson disease. Neurol Genet. 2020; 6(5): e498, doi: 10.1212/NXG.0000000000000498, indexed in Pubmed: 32802956.

12. Siuda J, Boczarska-Jedynak M, Budrewicz S, et al. Validation of the Polish version of the Movement Disorder Society-Unified Parkinson's Disease Rating Scale (MDS-UPDRS). Neurol Neurochir Pol. 2020; 54(5): 416-425, doi: 10.5603/PJNNS.a2020.0049, indexed in Pubmed: 32639019.

13. de Gr, Turleau C. Clinical Atlas of Human Chromosomes. Wiley Medical: New York. ; 1984.

14. Tezzon F, Zanoni T, Passarin MG, et al. Dystonia in a patient with deletion of 18p. Ital J Neurol Sci. 1998; 19(2): 90-93, doi: 10.1007/ BF02427563, indexed in Pubmed: 10935843.
15. Esposito F, Addor MC, Humm AM, et al. GNAL deletion as a probable cause of dystonia in a patient with the 18p- syndrome. Parkinsonism Relat Disord. 2014; 20(3): 351-352, doi: 10.1016/j.parkreldis.2013.12.005, indexed in Pubmed: 24405754.

16. Riva P, Ratti A, Venturin M. The Long Non-Coding RNAs in Neurodegenerative Diseases: Novel Mechanisms of Pathogenesis. Curr Alzheimer Res. 2016; 13(11): 1219-1231, doi: 10.2174/15672050136 66160622112234, indexed in Pubmed: 27338628.

17. Tonkin ET, Smith M, Eichhorn P, et al. A giant novel gene undergoing extensive alternative splicing is severed by a Cornelia de Lange-associated translocation breakpoint at 3q26.3. Hum Genet. 2004; 115(2): 139-148, doi: 10.1007/s00439-004-1134-6, indexed in Pubmed: 15168106.

18. Evers C, Mitter D, Strobl-Wildemann G, et al. Duplication Xp11.22-p14 in females: does $X$-inactivation help in assessing their significance? Am J Med Genet A. 2015; 167A(3): 553-562, doi: 10.1002/ ajmg.a.36897, indexed in Pubmed: 25691408.

19. Madrigal I, Fernández-Burriel M, Rodriguez-Revenga L, et al. Xq26.2-q26.3 microduplication in two brothers with intellectual disabilities: clinical and molecular characterization. J Hum Genet. 2010; 55(12): 822826, doi: 10.1038/jhg.2010.119, indexed in Pubmed: 20861843.

20. Wirth T, Mariani LL, Bergant G, et al. Loss-of-Function Mutations in NR4A2 Cause Dopa-Responsive Dystonia Parkinsonism. Mov Disord. 2020; 35(5): 880-885, doi: 10.1002/mds.27982, indexed in Pubmed: 31922365.

21. Palumbo O, Fischetto R, Palumbo P, et al. De novo microduplication of $\mathrm{CHL1}$ in a patient with non-syndromic developmental phenotypes. Mol Cytogenet. 2015; 8: 66, doi: 10.1186/s13039-015-0170-3, indexed in Pubmed: 26279679.

22. Vissers LE, Veltman JA, van Kessel AdG, et al. Identification of disease genes by whole genome CGH arrays. Hum Mol Genet. 2005; 14 Spec No. 2: R215-R223, doi: 10.1093/hmg/ddi268, indexed in Pubmed: 16244320.

23. Fiandaca MS, Lonser RR, Elder JB, et al. Advancing gene therapies, methods, and technologies for Parkinson's Disease and other neurological disorders. Neurol Neurochir Pol. 2020; 54(3): 220-231, doi: 10.5603/PJNNS.a2020.0046, indexed in Pubmed: 32557526. 